\title{
Dark energy fingerprints in the nonminimal Wu-Yang wormhole structure
}

\author{
Alexander B. Balakin ${ }^{1, *}$ and Alexei E. Zayats ${ }^{1,0}$ \\ ${ }^{1}$ Department of General Relativity and Gravitation, Institute of Physics, \\ Kazan Federal University, Kremlevskaya str. 18, Kazan 420008, Russia
}

\begin{abstract}
We discuss new exact solutions to nonminimally extended Einstein-Yang-Mills equations describing spherically symmetric static wormholes supported by the gauge field of the Wu-Yang type in a dark energy environment. We focus on the analysis of three types of exact solutions to the gravitational field equations. Solutions of the first type relate to the model, in which the dark energy is anisotropic, i.e., the radial and tangential pressures do not coincide. Solutions of the second type correspond to the isotropic pressure tensor; in particular, we discuss the exact solution, for which the dark energy is characterized by the equation of state for a string gas. Solutions of the third type describe the dark energy model with constant pressure and energy density. For the solutions of the third type, we consider in detail the problem of horizons and find constraints for the parameters of nonminimal coupling and for the constitutive parameters of the dark energy equation of state, which guarantee that the nonminimal wormholes are traversable.
\end{abstract}

PACS numbers: $04.20 . \mathrm{Jb}, 14.80 . \mathrm{Hv}, 04.20 . \mathrm{Gz}$

\section{INTRODUCTION}

Dark energy, the main constituent of a cosmic dark fluid, is considered nowadays as a key element of numerous cosmological models (see, e.g., [1] [4]). Originally, the term dark energy was introduced into the scientific lexicon in order to explain the discovery of the late-time accelerated expansion of the Universe [5 7]. However, there exist alternative explanations of this observational fact, for instance, in the framework of modified gravity (see, e.g., the review [8] for references and details). This means that we need to extend our knowledge about interactions of the dark energy with matter and fields in order to say definitely whether the dark energy is the real medium with exotic properties or the accelerated expansion of the Universe is the effective result of gravitational interactions with modified laws of attraction/repulsion. Clearly, there are dark energy interactions of two types: the indirect and direct ones. The first channel of indirect interaction with matter and fields is realized via the global gravity field; this channel is just visualized by the accelerated expansion of the Universe. The most known channel of direct interaction is presented by the coupling of the dark energy with dark matter, the second constituent of the dark fluid (see, e.g., [9 11]). The interactions of this type describe fine details of the cosmological expansion thus clarifying the fate of the Universe (big rip, little rip, pseudo-rip, etc.), solving the coincidence problem and answering the question of how many epochs of accelerated and decelerated expansion the Universe history includes. There are a few mathematical schemes of the description of the coupling between the dark energy and dark matter; for instance, in [12] we introduced the model force linear in the four-gradient of the dark energy pressure, which acts on the dark matter particles in analogy with the classical Archimedean force. The theory of direct dark energy interactions with scalar, pseu-

*Electronic address: Alexander.Balakin@kpfu.ru

†Electronic address: Alexei.Zayats@kpfu.ru doscalar, vector, electromagnetic, and gauge fields is less elaborated at present but seems to be very promising from the point of view of cosmological and astrophysical applications. For instance, in [13] studying the pyromagnetic, piezoelectric, and striction-type schemes of the direct interactions between the dark energy and electromagnetic field, we have found that specific unlighted epochs can appear in the Universe history. In other words, one can try to find some dark energy fingerprints in the Universe history, which are marked due to the coupling with electromagnetic fields.

In this paper we consider the nonminimal scheme of the indirect coupling of the dark energy to the gauge field. The model with an $\mathrm{SU}(\mathrm{N})$ symmetric gauge field is known to be indicated as the nonminimal one, if the Lagrangian of the model contains the so-called crossinvariants constructed as a tensorial product of the Riemann tensor, $R_{k m n}^{i}$, and its convolutions, on the one hand, and of the Yang-Mills field strength tensor, $F_{i k}^{(a)}$, on the other hand (see, e.g., 14 16 for references). In order to interpret the role of the dark energy in such models, let us focus on the examples of static spherically symmetric solutions to the nonminimally extended Einstein-Yang-Mills equations (see [16 18] for details). When the dark energy is considered to be absent, we refer to the exact solutions describing regular nonminimal Wu-Yang monopoles and wormholes of a magnetic type [17, 18] as well as traversable nonminimal wormholes of an electric type [19]. When the dark energy appears as the third player in the nonminimal model, we intend to focus on the search for dark energy fingerprints in the causal structure of the mentioned spherically symmetric static objects. To be more precise, in this paper, we focus on exact solutions of the nonminimally extended Einstein-Yang-Mills model, which describe nonminimal wormholes supported by Yang-Mills field in the dark energy environment.

Let us mention that in the cosmological context the dark energy is usually considered as a spatially homogeneous substrate, the pressure and energy density of which depend on time only. The most known model of this class relates to the so-called $\Lambda$-representation of the dark energy, characterized by constant energy den- 
sity $W_{(\mathrm{DE})}=\frac{\Lambda}{8 \pi}$ and pressure $P_{(\mathrm{DE})}=-\frac{\Lambda}{8 \pi}$ (several examples of wormhole solutions with the $\Lambda$-term can be found, e.g., in [20-22]). However, when we deal with the dark energy influence on the throat structure of the spherically symmetric static nonminimal wormhole, it is natural to treat the dark energy as a static spatially inhomogeneous substrate, the pressure of which depends on the radial variable $r$ only. The corresponding approach is motivated mathematically in Subsection IIIB.

The paper is organized as follows. In Section II we formulate the six-parameter nonminimal EinsteinYang-Mills-dark energy model, and represent master equations for the gauge and gravitational fields. In Section III. we introduce key assumptions about the equation of state of the dark energy and describe the Wu-Yang ansatz for the structure of the gauge field. In Section IV, we present exact solutions of the wormhole type for various models of the dark energy, including the $\Lambda$-term-type configuration. In Section V], we discuss in detail the causal structure of the spacetime for the $\Lambda$-type case. Section $V I$ is devoted to discussions.

\section{NONMINIMAL MASTER EQUATIONS}

\section{A. The action functional}

We start with the action functional

$$
\begin{aligned}
S & =\int d^{4} x \sqrt{-g}\left[\frac{R}{16 \pi}+L_{(\mathrm{DE})}+\frac{1}{4} F_{i k}^{(a)} F^{i k(a)}\right. \\
& \left.+\frac{1}{4} \mathcal{R}^{i k m n} F_{i k}^{(a)} F_{m n}^{(a)}\right]
\end{aligned}
$$

where as usual $g=\operatorname{det}\left(g_{i k}\right)$ is the determinant of a metric tensor $g_{i k}, R$ is the Ricci scalar, the Latin indices without parentheses run from 0 to 3 . The term $L_{(\mathrm{DE})}$ is the Lagrangian describing the dark energy. The tensor of nonminimal susceptibility $\mathcal{R}^{i k m n}$ is defined as follows:

$$
\begin{aligned}
\mathcal{R}^{i k m n} & \equiv \frac{q_{1}}{2} R\left(g^{i m} g^{k n}-g^{i n} g^{k m}\right) \\
& +\frac{q_{2}}{2}\left(R^{i m} g^{k n}-R^{i n} g^{k m}+R^{k n} g^{i m}-R^{k m} g^{i n}\right) \\
& +q_{3} R^{i k m n},
\end{aligned}
$$

where $R^{i k}$ and $R^{i k m n}$ are the Ricci and Riemann tensors, respectively, and $q_{1}, q_{2}, q_{3}$ are the phenomenological parameters describing the nonminimal coupling of the Yang-Mills fields with gravitation. We consider the Yang-Mills fields taking the values in the Lie algebra of the gauge group $\mathrm{SU}(2)$, so that $A_{i}^{(a)}$ and $F_{m n}^{(a)}$ are the Yang-Mills field potential and strength, respectively, the group index $(a)$ runs from 1 to 3 . The YangMills fields $F_{m n}^{(a)}$ are connected with the potentials of the gauge field $A_{i}^{(a)}$ by the well-known formulas (see, e.g., [23])

$$
F_{m n}^{(a)}=\nabla_{m} A_{n}^{(a)}-\nabla_{n} A_{m}^{(a)}+f_{(b)(c)}^{(a)} A_{m}^{(b)} A_{n}^{(c)} .
$$

Here $\nabla_{m}$ is a covariant spacetime derivative, the symbols $f_{(a)(b)(c)} \equiv \varepsilon_{(a)(b)(c)}$ denote the real structure constants of the gauge group $\mathrm{SU}(2)$.
The nonminimal susceptibility tensors $\mathcal{R}^{i k m n}$ contains three phenomenological parameters $q_{1}, q_{2}$ and $q_{3}$. We consider these three parameters to be independent coupling constants, and this choice is historically motivated. In the pioneer work [24], Prasanna introduced only one phenomenological parameter in front of the Riemann tensor $R^{i k m n}$ in the new cross-term $q R^{i k m n} F_{i k} F_{m n}$ appeared in the nonminimally extended Lagrangian. Later, Drummond and Hathrell [25], using the one-loop corrections to QED, showed that the tensor $\mathcal{R}^{i k m n}$ possesses just the structure (2), with $q_{1}=-5 q, q_{2}=13 q, q_{3}=-2 q$, where the positive parameter $q \equiv \frac{\alpha \lambda_{\mathrm{e}}^{2}}{180 \pi}$ is constructed by using the fine structure constant $\alpha$ and the Compton wavelength of the electron $\lambda_{\mathrm{e}}$. In other words, direct calculations of Drummond and Hathrell have fixed attention on the fact that there are three different coupling nonminimal constants, $q_{1}, q_{2}$, and $q_{3}$, which are proportional to the one nonminimal parameter $q$ with the dimensionality of length in square. In the paper [26], a general EinsteinMaxwell model was studied, in the framework of which the coupling constants $q_{1}, q_{2}$ and $q_{3}$ are considered to be independent parameters. The motivation of this idea is based on the irreducible representation of the curvature tensor

$$
R^{i k m n}=\mathcal{C}^{i k m n}+\mathcal{E}^{i k m n}+\mathcal{G}^{i k m n},
$$

where $\mathcal{C}^{i k m n}$ is the traceless Weyl tensor, and the standard formulas are introduced:

$$
\begin{gathered}
\left.\mathcal{E}^{i k m n} \equiv \frac{1}{2}\left(\mathcal{S}^{i m} g^{k n}-\mathcal{S}^{i n} g^{k m}+\mathcal{S}^{k n} g^{i m}-\mathcal{S}^{k m} g^{i n}\right)\right) \\
\mathcal{S}^{m n} \equiv R^{m n}-\frac{1}{4} R g^{m n} \\
\mathcal{G}^{i k m n} \equiv \frac{1}{12} R\left(g^{i m} g^{k n}-g^{i n} g^{k m}\right) \\
\mathcal{C}^{m}{ }_{n m k}=0, \quad \mathcal{S}_{m}^{m}=0, \quad \mathcal{G}^{m n}{ }_{m n}^{m n}=R
\end{gathered}
$$

(we follow the notations from the book [27]). Since $\mathcal{C}^{i k m n}, \mathcal{E}^{i k m n}$, and $\mathcal{G}^{i k m n}$ are independent (irreducible) parts of the decomposition of the Riemann tensor, it is reasonable to represent the nonminimal susceptibility tensor as the sum of three independent parts:

$$
\mathcal{R}^{i k m n}=\lambda_{1} \mathcal{G}^{i k m n}+\lambda_{2} \mathcal{E}^{i k m n}+\lambda_{3} \mathcal{C}^{i k m n},
$$

with phenomenological constants $\lambda_{1}, \lambda_{2}$ and $\lambda_{3}$. Clearly, (6) converts into (2), when

$$
\lambda_{1}=6 q_{1}+3 q_{2}+q_{3}, \quad \lambda_{2}=q_{2}+q_{3}, \quad \lambda_{3}=q_{3} .
$$

In other words, we have two equivalent irreducible decompositions of the susceptibility tensor, (6) and (2), but we prefer to use (2), keeping in mind historical motives.

\section{B. Nonminimal extension of the Yang-Mills equations}

Variation of the action (11) with respect to the Yang-Mills potential $A_{i}^{(a)}$ yields

$$
\nabla_{k} H^{(a) i k}+f_{(b)(c)}^{(a)} A_{k}^{(b)} H^{(c) i k}=0 .
$$


The tensor $H^{(b) i k}=F^{(b) i k}+\mathcal{R}^{i k m n} F_{m n}^{(b)}$ is a nonAbelian analog of the excitation tensor, known in the electrodynamics [28, 29].

\section{Master equations for the gravitational field}

The variation of the action functional (1) with respect to metric yields

$$
R_{i k}-\frac{1}{2} R g_{i k}=8 \pi T_{i k}^{(\mathrm{eff})}
$$

The effective stress-energy tensor $T_{i k}^{(\mathrm{eff})}$ can be divided into five parts:

$$
\begin{gathered}
T_{i k}^{(\mathrm{eff})}=T_{i k}^{(\mathrm{DE})}+T_{i k}^{(\mathrm{YM})} \\
+q_{1} T_{i k}^{(I)}+q_{2} T_{i k}^{(I I)}+q_{3} T_{i k}^{(I I I)} .
\end{gathered}
$$

The first term

$$
T_{i k}^{(\mathrm{DE})} \equiv-\frac{2}{\sqrt{-g}} \frac{\delta\left[\sqrt{-g} L_{(\mathrm{DE})}\right]}{\delta g^{i k}},
$$

describes the stress-energy tensor of the dark energy. As usual, we assume that this tensor possesses timelike eigen-four-vector $U^{i}$ normalized by unity and denote the corresponding eigenvalue as $W$, i.e., we assume that

$$
T_{i k}^{(\mathrm{DE})} U^{k}=W U_{i}, \quad g_{i k} U^{i} U^{k}=1 .
$$

The other three eigenvalues are denoted as $\Pi_{1}, \Pi_{2}$, and $\Pi_{3}$. The second term

$$
T_{i k}^{(\mathrm{YM})} \equiv \frac{1}{4} g_{i k} F_{m n}^{(a)} F^{m n(a)}-F_{i n}^{(a)} F_{k}^{n(a)}
$$

is a stress-energy tensor of the pure Yang-Mills field. The last three terms in (10) describe nonminimal contributions into the stress-energy tensor. Since the parameters $q_{1}, q_{2}$, and $q_{3}$ are independent coupling constants appearing in the irreducible representation of the susceptibility tensor $\mathcal{R}^{i k m n}$ (2), we decomposed this nonminimal contribution into the stress-energy tensor as a sum of three terms with $q_{1}, q_{2}$, and $q_{3}$ in front of the following tensors:

$$
\begin{aligned}
T_{i k}^{(I)} & =R T_{i k}^{(Y M)}-\frac{1}{2} R_{i k} F_{m n}^{(a)} F^{m n(a)} \\
& +\frac{1}{2}\left[\hat{D}_{i} \hat{D}_{k}-g_{i k} \hat{D}^{l} \hat{D}_{l}\right]\left[F_{m n}^{(a)} F^{m n(a)}\right], \quad(14) \\
T_{i k}^{(I I)}= & -\frac{1}{2} g_{i k}\left[\hat{D}_{m} \hat{D}_{l}\left(F^{m n(a)} F_{n}^{l(a)}\right)\right. \\
- & \left.R_{l m} F^{m n(a)} F_{n}^{l}(a)\right]-F^{l n(a)}\left(R_{i l} F_{k n}^{(a)}+R_{k l} F_{i n}^{(a)}\right) \\
- & R^{m n} F_{i m}^{(a)} F_{k n}^{(a)}-\frac{1}{2} \hat{D}^{m} \hat{D}_{m}\left(F_{i n}^{(a)} F_{k}^{n(a)}\right) \\
+ & \frac{1}{2} \hat{D}_{l}\left[\hat{D}_{i}\left(F_{k n}^{(a)} F^{l n(a)}\right)+\hat{D}_{k}\left(F_{i n}^{(a)} F^{l n(a)}\right)\right],
\end{aligned}
$$

$$
\begin{aligned}
T_{i k}^{(I I I)} & =\frac{1}{4} g_{i k} R^{m n l s} F_{m n}^{(a)} F_{l s}^{(a)} \\
& -\frac{3}{4} F^{l s(a)}\left(F_{i}^{n(a)} R_{k n l s}+F_{k}^{n(a)} R_{i n l s}\right) \\
& -\frac{1}{2} \hat{D}_{m} \hat{D}_{n}\left[F_{i}^{n(a)} F_{k}^{m(a)}+F_{k}^{n(a)} F_{i}^{m(a)}\right] .
\end{aligned}
$$

We use the following rule:

$$
\begin{aligned}
\hat{D}_{m} Q_{\cdots(d)}^{(a) \cdots} & \equiv \nabla_{m} Q_{\cdots(d)}^{(a) \cdots}+f_{\cdot(b)(c)}^{(a)} A_{m}^{(b)} Q_{\cdots(d)}^{(c) \cdots}+\ldots \\
& -f_{\cdot(b)(d)}^{(c)} A_{m}^{(b)} Q_{\cdots(c)}^{(a) \cdots}-\ldots
\end{aligned}
$$

for the derivative of the arbitrary tensor defined in the group space [30].

\section{Compatibility conditions}

The Bianchi identities require the total stressenergy tensor to be divergence-free, i.e., $\nabla^{k} T_{i k}^{(\mathrm{eff})}=0$. Using the decomposition (10)-(17) and the Yang-Mills field equations (8) one can show explicitly that this compatibility condition reduces to the requirement $\nabla^{k} T_{i k}^{(\mathrm{DE})}=0$, i.e., we deal with a separate conservation law for the dark energy. When all four eigenvalues, $W, \Pi_{1}, \Pi_{2}$, and $\Pi_{3}$ coincide, one obtains that $T_{i k}^{(\mathrm{DE})}=\frac{\Lambda}{8 \pi} g_{i k}$, and the compatibility conditions yield $\nabla_{i} \Lambda=0$, i.e., $\Lambda$ is a constant. In other words, the case when the dark energy can be presented in terms of cosmological constant,

$$
W=\frac{\Lambda}{8 \pi}, \quad \Pi_{1}=\Pi_{2}=\Pi_{3}=-P=\frac{\Lambda}{8 \pi}=W,
$$

is also included into our scheme of analysis.

\section{NONMINIMAL WORMHOLE OF THE WU-YANG TYPE IN THE DARK ENERGY ENVIRONMENT}

In order to model a traversable wormhole as a bridge joining two different spacetimes, one needs to have specific "bricks" possessing exotic properties, which guarantee violation of the null energy condition in the wormhole throat 31, 32]. The history of investigations in the wormhole sector of field theory can be found in [33, 34]; we attract the attention of the reader to the two episodes only. In 1973, Ellis [35] and, independently, Bronnikov 36] constructed wormhole solutions in the framework of the Einstein theory of gravity with a scalar field, which has negatively defined kinetic energy (phantom field in modern terminology). This first example can be indicated, indeed, as the exotic one from the point of view of classical field theory. The second example is connected with the work of Barcelló and Visser [37], who have shown that an ordinary (standard) scalar field can support a wormhole structure if this scalar field is nonminimally coupled to the spacetime curvature. These two examples illustrate the typical alternative: in order to justify the wormhole existence one can either provide exotic properties of the "bricks" 
for its throat or admit that the "bricks" are ordinary, but they interact nonminimally with spacetime curvature. We use the second way for modeling the wormhole configurations. For instance, in [19] one can find exact solutions for a traversable wormhole supported by an electric field nonminimally coupled to curvature. In the paper [18], we reconstructed the wormhole of the $\mathrm{Wu}$ Yang type supported by $\mathrm{SU}(2)$ symmetric gauge field of the magnetic type nonminimally coupled to gravity. Since the model that we study now is a natural extension of that model, let us comment briefly three key-elements of the wormhole-type solutions with a $\mathrm{Wu}-$ Yang-type gauge field nonminimally coupled to gravity in case when the interactions with dark energy are absent.

First of all, from our point of view the gauge fields attract special attention in the context of wormhole modeling: on the one hand, the Yang-Mills field is the most known contributor into the modern High-Energy Physics models; on the other hand, the Yang-Mills potentials form $\mathrm{SU}(\mathrm{N})$ multiplets, thus providing a versatile instrument for theoretical modeling.

Second, the pure Yang-Mills field itself is nonexotic, since it cannot violate the null energy condition in the wormhole throat, and, therefore, known wormhole solutions require either an additional phantom scalar field [38] or a surgery technique 39]; however, being coupled nonminimally to the spacetime curvature, the Yang-Mills field becomes able to organize a traversable wormhole throat (see [18]).

Third, the traversability of the Wu-Yang wormhole and the value of the radius of the throat can be the subject of tuning: depending on the relationships between the principal nonminimal coupling constant $q$ and the magnetic charge $\nu$, the throat happens to be closed or opened (see [18] for details). The following question is of great interest: physical processes of what type could regulate the traversability of the nonminimal Wu-Yang wormhole? The results of the paper 18] show that when the charge $\nu$ grows horizons appear and the wormhole becomes nontraversable (the cosmic gates happen to be closed). When the coupling constant $q$ increases, the cosmic gates, per contra, become opened. As it will be shown below, when we consider an additional element of the theoretical modeling, the dark energy, we obtain a new possibility for modeling the cosmic gates opening and closing.

\section{A. Wu-Yang type solution to the Yang-Mills field equations}

Let us consider a static spherically symmetric spacetime with the metric

$$
\begin{gathered}
d s^{2}=\sigma^{2} N d t^{2}-\frac{d r^{2}}{N}-R^{2}(r)\left(d \theta^{2}+\sin ^{2} \theta d \varphi^{2}\right) \\
r \in(-\infty ;+\infty)
\end{gathered}
$$

Here $\sigma, N$, and $R(r)$ are functions depending on the radial coordinate $r$ only. Since the functions, which characterize the dark energy, do not enter the master equations for the gauge field, we obtain immediately that, as in the cases of a nonminimal SU(2) monopole [17] and wormhole [18], the special ansatz (see [40, 41]),

$$
\mathbf{A}_{0}=\mathbf{A}_{r}=0, \quad \mathbf{A}_{\theta}=i \mathbf{t}_{\varphi}, \quad \mathbf{A}_{\varphi}=-i \nu \sin \theta \mathbf{t}_{\theta},
$$

gives the exact solution with the field strength tensor of the following form:

$$
\mathbf{F}_{i k}=\delta_{i}^{\theta} \delta_{k}^{\varphi} \mathbf{F}_{\theta \varphi}, \quad \mathbf{F}_{\theta \varphi}=i \nu \sin \theta \mathbf{t}_{r}
$$

The parameter $\nu$ is an integer and it denotes a magnetic charge. Let us remind that the generators $\mathbf{t}_{r}, \mathbf{t}_{\theta}$ and $\mathbf{t}_{\varphi}$ are the position-dependent ones and are connected with the standard generators of the $\mathrm{SU}(2)$ group as

$$
\begin{array}{r}
\mathbf{t}_{r}=\cos \nu \varphi \sin \theta \mathbf{t}_{(1)}+\sin \nu \varphi \sin \theta \mathbf{t}_{(2)}+\cos \theta \mathbf{t}_{(3)}, \\
\mathbf{t}_{\theta}=\partial_{\theta} \mathbf{t}_{r}, \quad \mathbf{t}_{\varphi}=\frac{1}{\nu \sin \theta} \partial_{\varphi} \mathbf{t}_{r} ;
\end{array}
$$

they satisfy the relations

$$
\left[\mathbf{t}_{r}, \mathbf{t}_{\theta}\right]=i \mathbf{t}_{\varphi}, \quad\left[\mathbf{t}_{\theta}, \mathbf{t}_{\varphi}\right]=i \mathbf{t}_{r}, \quad\left[\mathbf{t}_{\varphi}, \mathbf{t}_{r}\right]=i \mathbf{t}_{\theta}
$$

The system of Yang-Mills equations (8) is satisfied identically for arbitrary curvature tensor and for arbitrary equation of state of the dark energy.

Again, this solution is effectively Abelian, i.e., by the suitable gauge transformation $\mathbf{U}=\exp \left(-i \theta \mathbf{t}_{\varphi}\right)$ it can be converted into the product of the Dirac-type potential and the gauge group generator $\mathbf{t}_{(3)}$.

\section{B. Assumptions about the dark energy}

The simplest variant to introduce the dark energy is known to connect with cosmological $\Lambda$ term. In the de Sitter spacetime with the so-called $t$-representation of the metric

$$
d s^{2}=d t^{2}-a^{2}\left(t_{0}\right) e^{2 \sqrt{\frac{\Lambda}{3}}\left(t-t_{0}\right)}\left(d r^{2}+r^{2} d \Omega^{2}\right),
$$

the dark energy pressure and energy-density scalar are constant and they can be written, respectively, in the form

$$
\begin{gathered}
P_{(\mathrm{DE})}=-\frac{\Lambda}{8 \pi}, \quad W_{(\mathrm{DE})}=\frac{\Lambda}{8 \pi}, \\
P_{(\mathrm{DE})}+W_{(\mathrm{DE})}=0 .
\end{gathered}
$$

In more sophisticated models these quantities are considered to be functions of cosmological time $P_{(\mathrm{DE})}(t)$, $W_{(\mathrm{DE})}(t)$, nevertheless, the specific equation of state with $P_{(\mathrm{DE})}(t)+W_{(\mathrm{DE})}(t) \leq 0$ remains the distinguishing feature.

The de Sitter spacetime can also be described in the so-called $r$-representation using the equivalent metric

$$
d s^{2}=\left(1-\frac{\Lambda r^{2}}{3}\right) d t^{2}-\left(1-\frac{\Lambda r^{2}}{3}\right)^{-1} d r^{2}-r^{2} d \Omega^{2}
$$

which, clearly, is static and depends on the radial variable $r$ only. The corresponding Schwarzschild-ReissnerNordström, etc., extensions of the model lead to the 
replacements

$$
\begin{aligned}
\left(1-\frac{\Lambda r^{2}}{3}\right) & \rightarrow\left(1-\frac{\Lambda r^{2}}{3}-\frac{2 M}{r}+\frac{Q^{2}}{2 r^{2}}\right) \rightarrow \\
& \rightarrow\left(1-\frac{\Lambda r^{2}}{3}+f(r)\right),
\end{aligned}
$$

where $M$ is the mass and $Q$ is the charge of the object. Studying this model and more sophisticated models, we have a possibility to interpret the contributions containing $\Lambda$ in terms of dark energy of the $\Lambda$-type. Our ansatz is that one can develop this idea and consider the dark energy in the $r$-representation, i.e., using the functions $P_{(\mathrm{DE})}(r), W_{(\mathrm{DE})}(r)$ satisfying the typical condition $P_{(\mathrm{DE})}(r)+W_{(\mathrm{DE})}(r) \leq 0$.

For the static spherically symmetric configuration, the two diagonal components of the effective stressenergy tensor coincide, $T^{(\mathrm{eff})}{ }_{\theta}^{\theta}=T^{(\mathrm{eff})}{ }_{\varphi}^{\varphi}$. Based on this fact, we assume that two of the three eigenvalues $\Pi_{1}$, $\Pi_{2}, \Pi_{3}$ of the stress-energy tensor of the dark energy also coincide, thus providing the following definitions

$$
-P_{\|} \equiv T_{r}^{(\mathrm{DE})^{r},} \quad-P_{\perp} \equiv T_{\theta}^{(\mathrm{DE})^{\theta}}=T_{\varphi}^{(\mathrm{DE})^{\varphi}} .
$$

In other words we can introduce longitudinal, $P_{\|}$, and transversal, $P_{\perp}$ pressures, prescribed to the dark energy. We assume that the dark energy equations of state are linear

$$
P_{\|}=\omega_{\|} W, \quad P_{\perp}=\omega_{\perp} W .
$$

When the dark energy is considered to possess an isotropic pressure, we have to put $\omega_{\|}=\omega_{\perp}$.

\section{EXACT SOLUTIONS TO THE GRAVITATIONAL FIELD EQUATIONS}

\section{A. Key equations}

For the metric (19), only four components of the Einstein tensor $G_{i}^{k}=R_{i}^{k}-\frac{1}{2} \delta_{i}^{k} R$ are nonvanishing, $G_{0}^{0}$, $G_{r}^{r}$, and $G_{\theta}^{\theta}=G_{\varphi}^{\varphi}$. In order to describe the gravity field we use the following three independent equations. First, we consider the difference of the first two equations, $G_{0}^{0}-G_{r}^{r}=8 \pi\left(T^{(\text {eff })}{ }_{0}^{0}-T_{r}^{\left(\text {eff }{ }_{r}^{r}\right.}\right)$, yielding

$$
\begin{gathered}
\left(1-\frac{\kappa q_{1}}{R^{4}}\right)\left[\frac{\sigma^{\prime} R^{\prime}}{\sigma R}-\frac{R^{\prime \prime}}{R}\right] \\
=\frac{\kappa\left(10 q_{1}+4 q_{2}+q_{3}\right) R^{\prime 2}}{R^{6}}+\frac{4 \pi\left(W+P_{\|}\right)}{N} .
\end{gathered}
$$

Second, we use the Einstein equation $G_{0}^{0}=8 \pi T^{(\mathrm{eff})}{ }_{0}^{0}$, which gives

$$
\begin{aligned}
& \frac{1-N R^{\prime 2}}{R^{2}}-\left(1-\frac{\kappa q_{1}}{R^{4}}\right)\left(\frac{N^{\prime} R^{\prime}}{R}+\frac{2 N R^{\prime \prime}}{R}\right) \\
&= \frac{\kappa}{R^{4}}\left\{\frac{1}{2}-\frac{q_{1}+q_{2}+q_{3}}{R^{2}}+\frac{\left(13 q_{1}+4 q_{2}+q_{3}\right) N R^{\prime 2}}{R^{2}}\right\} \\
&+8 \pi W .
\end{aligned}
$$

Third, we consider the compatibility equation $\nabla^{k} T_{i k}^{(\mathrm{eff})}=0$, which can be reduced now to the equation of hydrostatic equilibrium

$$
P_{\|}^{\prime}+\frac{2 R^{\prime}}{R}\left(P_{\|}-P_{\perp}\right)+\left(W+P_{\|}\right) \frac{\left(\sigma^{2} N\right)^{\prime}}{2 \sigma^{2} N}=0 .
$$

The parameter $\kappa$ is defined as $\kappa=8 \pi \nu^{2}$. The prime denotes the derivative with respect to the variable $r$.

\section{Energy density distribution}

The equation (32) can be easily resolved using equations of state (29); this procedure yields

$$
\begin{aligned}
W(r) & =W_{0}\left[\frac{R(r)}{R(0)}\right]^{2 \alpha}\left[\sigma^{2}(r) N(r)\right]^{-\gamma}, \\
\alpha & \equiv \frac{\omega_{\perp}-\omega_{\|}}{\omega_{\|}}, \quad \gamma \equiv \frac{1+\omega_{\|}}{2 \omega_{\|}}
\end{aligned}
$$

where $R(0) \neq 0$ is the value of the radial function $R(r)$ at $r=0$ and $W_{0}$ is an integration constant with dimensionality of energy density. We have to stress, that the formula (33) describes the distribution of the energy density of the dark energy for arbitrary radial function $R(r)$ satisfying the condition $R(0) \neq 0$.

\section{Examples of the radial function $R(r)$}

The function $R(r)$ can be chosen according to physical requirements; when we consider the wormholetype solutions, we assume that

$$
R(0)=a>0, \quad R^{\prime}(0)=0, \quad R^{\prime \prime}(0)>0,
$$

where $a$ is the throat radius:

(1) The most known function satisfying these conditions is $R(r)=\sqrt{r^{2}+a^{2}}$; it is even with respect to the variable $r$. We will use it below for the reconstruction of exact solutions to the master equations with constant dark energy pressure; this radial function displays the asymptotic behavior $R(r \rightarrow \infty) \rightarrow r$.

(2) The conditions (34) are satisfied for the so-called catenary-type function $R(r)=a \cosh \frac{r}{a}$, which is also even function and has the asymptote $R(r \rightarrow \infty) \rightarrow a e^{\frac{r}{a}}$. This function satisfies the equation $R^{\prime \prime}=\frac{1}{a} R$ thus simplifying the equations (30) and (31). We will use this radial function below for the case of isotropic dark energy pressure with equation of state $P=-\frac{1}{3} W$.

(3) The equation (30) can be simplified essentially, when we consider $R(r)$ to satisfy the equation

$$
\left(1-\frac{\kappa q_{1}}{R^{4}}\right) \frac{R^{\prime \prime}}{R}=-\frac{\kappa\left(10 q_{1}+4 q_{2}+q_{3}\right) R^{\prime 2}}{R^{6}},
$$

which follows from (30) with $\sigma \equiv 1$ and $W+P_{||}=0$. The corresponding solution can be presented in quadratures as

$$
K r=\int_{a}^{R} d \xi \frac{\xi^{2}}{\sqrt{\xi^{4}-a^{4}}}
$$

and satisfies the conditions (34), when $12 q_{1}+4 q_{2}+q_{3}=0$, $q_{1}=\frac{a^{4}}{\kappa}>0$, and $K$ is arbitrary constant. 


\section{B. List of models: The reference model with absent dark energy}

When the dark energy is absent, the equations (30), (31) coincide with Eqs. (25), (27) of the paper [18], if we put $R(r)=\sqrt{r^{2}+a^{2}}, W=0$, and $P_{\|}=0$. Let us remind that in the case of reference model we deal with one-parameter family of exact solutions describing the wormhole with the throat radius $a$, when the following relationships between model parameters hold:

$$
q_{1}=\frac{a^{4}}{\kappa}, \quad q_{2}=-\frac{10 a^{4}}{3 \kappa}-\frac{a^{2}}{6}, \quad q_{3}=\frac{4 a^{4}}{3 \kappa}+\frac{2 a^{2}}{3} .
$$

In fact, the product of the nonminimal parameter $q_{1}$ and of the parameter $\kappa=8 \pi \nu^{2}$ predetermines the value of the throat radius, thus, the wormhole does not exist when $q_{1} \kappa=0$. Since the value of the metric function $N(r)$ at $r=0$ can be written as $N(0)=\frac{1}{3}\left(1-\frac{|\nu|}{\nu_{(\text {crit })}}\right)$ with $\nu_{\text {(crit) }} \equiv \sqrt{\frac{2 q_{1}}{\pi}}$, it is clear that $N(0)>0$ and the wormhole is traversable, when $|\nu|<\nu_{\text {(crit). When the }}$ gauge charge of the object, $\nu$, is changing (e.g., due to the charge loss) and becomes less than the critical value $\nu_{\text {(crit) }}$, the corresponding wormhole becomes traversable. In other words, the dimensionless parameter $\frac{\kappa}{4 a^{2}}=\frac{|\nu|}{\nu_{\text {(crit) }}}$ is the guiding parameter of the model. Below we will refer to these results discussing new features of the model under consideration.

\section{List of models: The first model with $\omega_{\|}=-1$}

When $\omega_{\|}=-1$, i.e., $W+P_{\|}=0$, the equation (30) does not contain the metric function $N(r)$, and thus converts into the equation for the function $\sigma$ only. Direct integration gives us $\sigma$ as a function of $R(r)$ and its derivative as follows:

$$
\sigma=\sigma_{0} R^{\prime}\left(\frac{R^{4}-\kappa q_{1}}{R^{4}}\right)^{\beta}, \quad \beta \equiv \frac{10 q_{1}+4 q_{2}+q_{3}}{4 q_{1}} .
$$

We are interested in the analysis of regular solutions for $\sigma(r)$. The first regularity requirement directly follows from (30) (supplemented by the relationship $\left.W+P_{\|}=0\right)$ : since $R^{\prime}(0)=0$ but $R^{\prime \prime}(0) \neq 0$, we have to put $\left(1-\frac{\kappa q_{1}}{R^{4}(0)}\right)=0$. This regularity requirement fixes the nonminimal parameter $q_{1}$ : first, it has to be positive, $q_{1}>0$; second, it has to be connected with the throat radius $a$ as $R(0)=a=\left(\kappa q_{1}\right)^{\frac{1}{4}}$. The second regularity requirement can be explained as follows. When the throat conditions (34) are satisfied, one obtains that $R(r \rightarrow 0) \rightarrow a+\frac{1}{2} R^{\prime \prime}(0) r^{2}$, and $R^{\prime}(r \rightarrow 0) \rightarrow R^{\prime \prime}(0) r$. This means that the function $\sigma(r)$ is regular at $r=0$, if and only if $1+2 \beta=0$, i.e., when $12 q_{1}+4 q_{2}+q_{3}=0$. As a result, we obtain the regular solution for the metric function:

$$
\sigma(r)=\sigma_{0} \frac{R^{\prime} R^{2}}{\sqrt{R^{4}-a^{4}}}, \quad \sigma(0)=\sigma_{0} \sqrt{\frac{a}{2} R^{\prime \prime}(0)} .
$$

As for the integration constant $\sigma_{0}$, if we assume that $R^{\prime}(r \rightarrow \infty) \rightarrow 1$, we can put $\sigma_{0}=1$, providing $\sigma(\infty)=1$.
The equation (31), clearly, admits the solution with finite value $N(0)$, when

$$
\frac{1}{R^{2}(0)}=\frac{\kappa}{R^{4}(0)}\left[\frac{1}{2}-\frac{q_{1}+q_{2}+q_{3}}{R^{2}(0)}\right]+8 \pi W_{0} .
$$

Thus, regularity of the metric functions $\sigma(r)$ and $N(r)$ is possible, when three nonminimal coupling parameters $q_{1}, q_{2}$, and $q_{3}$ are connected with the wormhole throat radius $R(0)=a$ by the following relationships:

$$
\begin{gathered}
q_{1}=\frac{a^{4}}{\kappa}, \quad q_{2}=-\frac{10 a^{4}}{3 \kappa}-\frac{a^{2}}{6}-\frac{8 \pi W_{0}}{3 \kappa} a^{6} \\
q_{3}=\frac{4 a^{4}}{3 \kappa}+\frac{2 a^{2}}{3}+\frac{32 \pi W_{0}}{3 \kappa} a^{6}
\end{gathered}
$$

When the dark energy is absent, i.e., $W_{0}=0$, these formulas recover the relationships (37) for the nonminimal Wu-Yang wormhole obtained in [18].

Taking into account (41), we obtain immediately the metric function $N(r)$ in the form

$$
\begin{gathered}
N(r)=\frac{R}{R^{\prime 2}(r) \sqrt{R^{4}-a^{4}}} \int_{0}^{r} \frac{R^{\prime}(x) d x}{R^{2} \sqrt{R^{4}-a^{4}}}\left\{R^{4}-a^{4}\right. \\
\left.-\frac{\kappa\left(R^{2}-a^{2}\right)}{2}-8 \pi W_{0} a^{6}\left[\left(\frac{R}{a}\right)^{4-2 \omega_{\perp}}-1\right]\right\} .
\end{gathered}
$$

The value $N(0)$ is finite; it is positive when

$$
a R^{\prime \prime}(0) N(0)=\frac{1}{3}-\frac{\kappa}{12 a^{2}}+\frac{4 \pi}{3} W_{0}\left(\omega_{\perp}-2\right) a^{2}>0 .
$$

There are four interesting subcases.

(i) $\omega_{\perp}=-1$ :

It is the subcase, when $P_{\perp}=P_{\|}=-W=-W_{0}$, and thus the scalars of the energy and pressure of the dark energy are constant. We deal now with the effective cosmological constant connected with $W_{0}$ by the relation $\Lambda=8 \pi W_{0}$. The metric function $N(r)$ simplifies

$$
\begin{aligned}
N(r) & =\frac{R(r)}{R^{\prime 2}(r) \sqrt{R^{4}-a^{4}}} \int_{0}^{r} \frac{R^{\prime}(x) d x}{R^{2}(x)} \sqrt{\frac{R^{2}-a^{2}}{R^{2}+a^{2}}} \\
& \times\left[\left(R^{2}+a^{2}\right)\left(1-\Lambda R^{2}\right)-\left(\frac{\kappa}{2}+\Lambda a^{4}\right)\right],
\end{aligned}
$$

nevertheless, the integral cannot be expressed in elementary functions; graphs, which illustrate the behavior of the functions of this type, will be discussed below. Since now

$$
a R^{\prime \prime}(0) N(0)=\frac{1}{3}-\frac{\kappa}{12 a^{2}}-\frac{\Lambda a^{2}}{2},
$$

the wormhole is traversable, when

$$
N(0)>0 \Rightarrow \Lambda<\frac{2}{3 a^{2}}\left(1-\frac{\kappa}{4 a^{2}}\right) .
$$

(ii) $\omega_{\perp}=0$ : 
In this subcase, the dark energy manifests dust properties in the tangential directions, $P_{\perp}=0$. The corresponding metric function $N(r)$ is

$$
\begin{aligned}
N(r) & =\frac{R(r)}{R^{\prime 2}(r) \sqrt{R^{4}-a^{4}}} \int_{0}^{r} \frac{R^{\prime}(x) d x}{R^{2}(x)} \sqrt{\frac{R^{2}-a^{2}}{R^{2}+a^{2}}} \\
& \times\left[\left(R^{2}+a^{2}\right)\left(1-8 \pi W_{0} a^{2}\right)-\frac{\kappa}{2}\right] .
\end{aligned}
$$

and the traversability condition $N(0)>0$ gives

$$
8 \pi W_{0}<\frac{1}{a^{2}}\left(1-\frac{\kappa}{4 a^{2}}\right) .
$$

(iii) $\omega_{\perp}=1$ :

In this case, the dark energy behaves as a stiff matter in the tangential directions, and we obtain

$$
\begin{aligned}
N(r) & =\frac{R(r)}{R^{\prime 2}(r) \sqrt{R^{4}-a^{4}}} \int_{0}^{r} \frac{R^{\prime}(x) d x}{R^{2}(x)} \sqrt{\frac{R^{2}-a^{2}}{R^{2}+a^{2}}} \\
& \times\left[\left(R^{2}+a^{2}\right)-\left(\frac{\kappa}{2}+8 \pi W_{0} a^{4}\right)\right] .
\end{aligned}
$$

thus the traversability condition yields

$$
8 \pi W_{0}<\frac{2}{a^{2}}\left(1-\frac{\kappa}{4 a^{2}}\right) \text {. }
$$

(iv) $\omega_{\perp}=2$ :

For this very special case, the parameter $W_{0}$ is hidden,

$$
\begin{aligned}
N(r) & =\frac{R}{R^{\prime 2}(r) \sqrt{R^{4}-a^{4}}} \int_{0}^{r} \frac{R^{\prime}(x) d x}{R^{2}} \sqrt{\frac{R^{2}-a^{2}}{R^{2}+a^{2}}} \times \\
& \times\left(R^{2}+a^{2}-\frac{\kappa}{2}\right),
\end{aligned}
$$

and the traversability condition reads $a^{2}>\frac{\kappa}{4}$.

\section{List of models: The second model with $\omega_{\|}=\omega_{\perp}=-\frac{1}{3}$}

This model relates to the isotropic dark energy with the equation of state $W+3 P=0$; such model was indicated in 42] as a "string gas". In the cosmological context the condition $W+3 P=0$ leads to the requirement that the acceleration parameter $q(t)$ is equal to zero identically, and the scale factor is a linear function of the cosmological time. Let us study this model in the context of the wormhole structure analysis.

When $P=-\frac{1}{3} W$, the energy density of the dark energy is distributed according to the formula

$$
W(r)=W_{0} \sigma^{2} N
$$

and the equation (30) for the metric function $\sigma(r)$ happens to be decoupled from the equation (31), which describes the metric function $N(r)$. Indeed, the equation (30) converts now to the Bernoulli differential equation:

$$
\begin{aligned}
\sigma^{\prime} & =\frac{\sigma}{R R^{\prime}\left(R^{4}-\kappa q_{1}\right)}\left[R R^{\prime \prime}\left(R^{4}-\kappa q_{1}\right)\right. \\
& \left.+\left(10 q_{1}+4 q_{2}+q_{3}\right) \kappa R^{\prime 2}+\frac{8 \pi}{3} W_{0} R^{6} \sigma^{2}\right],
\end{aligned}
$$

the formal solution to which has the form

$$
\begin{aligned}
\sigma & =R^{\prime}\left(1-\frac{\kappa q_{1}}{R^{4}}\right)^{\beta} \times \\
& \times\left[C-\frac{16 \pi W_{0}}{3} \int d r R R^{\prime}\left(1-\frac{\kappa q_{1}}{R^{4}}\right)^{(2 \beta-1)}\right]^{-1 / 2}, \\
\beta & =\frac{10 q_{1}+4 q_{2}+q_{3}}{4 q_{1}} .
\end{aligned}
$$

In this paper, we discuss (as an illustration) only one regular solution of this type with requirements $q_{1}=0$, $4 q_{2}+q_{3}=0$ and $\frac{8 \pi}{3} W_{0} a^{2}+1=0$. The last condition relates to the negative effective cosmological constant. This special solution is characterized by $\sigma(r) \equiv 1$ and the radial function $R(r)$, which describes the well-known catenary curve:

$$
R(r)=a \cosh \frac{r}{a}, R(0)=a, R^{\prime}(0)=0, R^{\prime \prime}(0)=\frac{1}{a} .
$$

The metric function $N(r)$ is now the solution to the linear equation

$$
\begin{aligned}
N^{\prime} & =-\frac{N}{R^{\prime} R}\left[2 R^{\prime \prime} R+R^{\prime 2}+8 \pi W_{0} R^{2}\right] \\
& -\frac{1}{2 R^{\prime} R^{5}}\left[6 \kappa q_{2}-2 R^{4}+\kappa R^{2}\right],
\end{aligned}
$$

it can be presented as the polynomial

$$
\begin{aligned}
N(z) & =\left[\frac{3 \kappa q_{2}}{a^{4}}+\frac{\kappa}{2 a^{2}}-1\right]+z C+ \\
& +z^{2}\left[\frac{6 \kappa q_{2}}{a^{4}}+\frac{\kappa}{2 a^{2}}\right]-z^{4} \frac{\kappa q_{2}}{a^{4}},
\end{aligned}
$$

where $z=\tanh \frac{r}{a}$ and $C$ is an integration constant. Let us mention that $N(-r)=N(r)$, when $C=0$. If, in addition, we choose the nonminimal coupling constant $q_{2}$ according to the relationship

$$
\frac{4 \kappa q_{2}}{a^{4}}=1-\frac{\kappa}{2 a^{2}},
$$

we obtain that $N(r= \pm \infty)=1$ and $N(0)=\frac{1}{4}\left(\frac{\kappa}{2 a^{2}}-1\right)$. In other words, the regular wormhole with a catenarytype throat filled by the isotropic dark energy with the equation of state $P=-\frac{1}{3} W$ is traversable, when $\kappa>2 a^{2}$.

\section{CAUSAL STRUCTURE OF WU-YANG WORMHOLES WITH DARK ENERGY OF THE $\Lambda$-TYPE}

\section{A. Search for horizons by the method of auxiliary function}

Let us consider the model with isotropic dark energy characterized by the following equations of state:

$$
P_{\|}=P_{\perp}=-W=-W_{0}=-\frac{\Lambda}{8 \pi} .
$$

Clearly, it is the case, which can be reduced to the nonminimal Einstein-Yang-Mills model with cosmological constant $\Lambda$. This submodel of the general model (of 
the wormhole filled with the dark energy) is chosen to analyze in more details the problem of horizons. To be more precise, we are interested to know how many horizons the nonminimal $\mathrm{Wu}$-Yang wormhole has and where these horizons appear. For this purpose we fix the radial function as

$$
R(r)=\sqrt{r^{2}+a^{2}}
$$

so that the regular metric function (39) takes the form

$$
\sigma(r)=\sqrt{\frac{r^{2}+a^{2}}{r^{2}+2 a^{2}}}, \quad \sigma(\infty)=1, \quad \sigma(0)=\frac{1}{\sqrt{2}} .
$$

The function $\sigma(r)$ reaches neither zero nor infinite values, thus, the causal structure of the wormhole is predetermined only by the properties of the function $N(r)$, which can be written as follows (see (44)):

$$
\begin{gathered}
N(r)=\frac{\left(r^{2}+a^{2}\right)^{3 / 2}}{r^{3} \sqrt{r^{2}+2 a^{2}}} \int_{0}^{r} \frac{x^{2} d x}{\left(x^{2}+a^{2}\right)^{3 / 2} \sqrt{x^{2}+2 a^{2}}} \times \\
\times\left[-\Lambda x^{4}+x^{2}\left(1-3 \Lambda a^{2}\right)+\left(2 a^{2}-\frac{\kappa}{2}-3 \Lambda a^{4}\right)\right], \\
N(0)=\frac{1}{3}-\frac{\kappa}{12 a^{2}}-\frac{\Lambda a^{2}}{2} .
\end{gathered}
$$

Clearly, we deal with three-parameter family of regular solutions: the metric function $N$ depends on the cosmological constant $\Lambda$, on the gauge charge $\kappa \equiv 8 \pi \nu^{2}$, and on the parameter of the nonminimal coupling $q_{1}$ through the throat radius $a \equiv\left(\kappa q_{1}\right)^{\frac{1}{4}}$. Horizons are known to appear at $r=r_{(\mathrm{s})}$, where $r_{(\mathrm{s})}$ are the zeroes of the metric function, i.e., $N\left(r_{(\mathrm{s})}\right)=0$. It is convenient to introduce the dimensionless variable $\xi=\frac{r}{a}$ and to rewrite (62) as

$$
N(\xi)=\frac{\left(\xi^{2}+1\right)^{3 / 2}}{\xi^{3} \sqrt{\xi^{2}+2}} I_{3}(\xi)\left[f\left(\xi, \frac{\kappa}{a^{2}}\right)-\Lambda a^{2}\right] .
$$

Here, we introduce the auxiliary function

$$
f\left(\xi, \frac{\kappa}{a^{2}}\right) \equiv \frac{I_{2}(\xi)}{I_{3}(\xi)}-\frac{\kappa}{2 a^{2}} \frac{I_{1}(\xi)}{I_{3}(\xi)},
$$

based on the integrals

$$
\begin{aligned}
& I_{1}(\xi)=\int_{0}^{\xi} \frac{x^{2} d x}{\left(x^{2}+1\right)^{3 / 2} \sqrt{x^{2}+2}}, \\
& I_{2}(\xi)=\int_{0}^{\xi} \frac{x^{2}\left(x^{2}+2\right) d x}{\left(x^{2}+1\right)^{3 / 2} \sqrt{x^{2}+2}}, \\
& I_{3}(\xi)=\int_{0}^{\xi} \frac{x^{2}\left(x^{4}+3 x^{2}+3\right) d x}{\left(x^{2}+1\right)^{3 / 2} \sqrt{x^{2}+2}} .
\end{aligned}
$$

These integrals do not include parameters; besides, all three functions $I_{1}(\xi), I_{2}(\xi), I_{3}(\xi)$ are odd functions of $\xi$, and they grow monotonically for $0<\xi<\infty$. The auxiliary function $f\left(\xi, \frac{\kappa}{a^{2}}\right)$ is even with respect to the variable $\xi$, and possesses the following asymptotical properties:

$$
f\left(\xi \rightarrow 0, \frac{\kappa}{a^{2}}\right)=\frac{2}{3}\left(1-\frac{\kappa}{4 a^{2}}\right) \equiv f(0),
$$

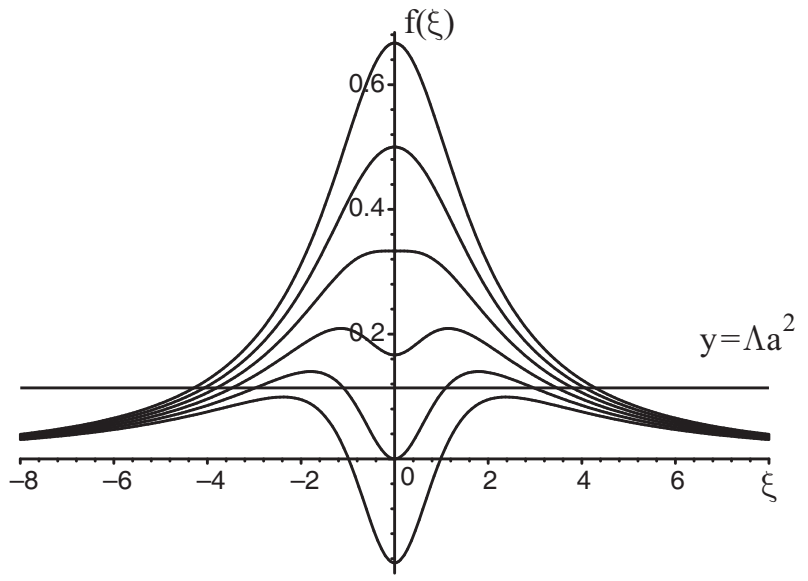

FIG. 1: Plots of the function $f\left(\xi, \frac{\kappa}{a^{2}}\right)$ for six values of the parameter $\frac{\kappa}{a^{2}}: 0,1, \ldots, 5$. The upper curve relates to the minimal value of the guiding parameter, $\frac{\kappa}{a^{2}}=0$; the maximal value of the function is $f(0,0)=\frac{2}{3}$; this curve plays the role of separatrix. When $\frac{\kappa}{a^{2}}=4, f(0,4)=0$ according to (69). The lower curve corresponds to the value $\frac{\kappa}{a^{2}}=5$. When $\frac{\kappa}{a^{2}} \leq 2$, the graphs have only one extremum (maximum) at $\xi=0$. When $\frac{\kappa}{a^{2}}>2$, two symmetric maxima and one minimum (at $\xi=0$ ) appear in the graphs. When the parameter $\frac{\kappa}{a^{2}}$ increases, the maxima drift to the left and right, respectively, and the minimal values behave as $f\left(0, \frac{\kappa}{a^{2}} \rightarrow \infty\right) \rightarrow-\infty$.

$$
f\left(\xi \rightarrow \infty, \frac{\kappa}{a^{2}}\right) \sim \frac{3}{\xi^{3}} \rightarrow 0 .
$$

The equation $N=0$, which determines the horizons, reduces now to the equation

$$
f\left(\xi, \frac{\kappa}{a^{2}}\right)=\Lambda a^{2},
$$

thus illustrating the fact that only two effective dimensionless parameters, $\frac{\kappa}{a^{2}}$ and $\Lambda a^{2}$, predetermine the structure of horizons. Typical plots of the function $f\left(\xi, \frac{\kappa}{a^{2}}\right)$ for several values of the dimensionless parameter $\frac{\kappa}{a^{2}}$ are presented in Fig. 1.

In order to find the number of horizons, one can determine the number of points in which the horizontal straight line $y=\Lambda a^{2}$ crosses the graph of the function $y=f\left(\xi, \frac{\kappa}{a^{2}}\right)$. Clearly, depending on the values of the parameters $\frac{\kappa}{a^{2}}$ and $\Lambda a^{2}$, zero, one, two, three or four cross-points can appear. Let us discuss this feature in more details.

\section{B. The structure of horizons}

In order to simplify the analysis, we distinguish three cases, which correspond to the following values of the guiding parameter $\frac{\kappa}{a^{2}}$ : first, $0 \leq \frac{\kappa}{a^{2}} \leq 2$, second, $2<\frac{\kappa}{a^{2}} \leq 4$, third, $\frac{\kappa}{a^{2}}>4$.

\section{The first case: $0 \leq \frac{\kappa}{a^{2}} \leq 2$}

For these values of the guiding parameter $\frac{\kappa}{a^{2}}$ the graph of the function $y=f\left(\xi, \frac{\kappa}{a^{2}}\right)$ lies at $y>0$ and it 
has one maximum at $\xi=0$, namely, $f_{\max }=f(0)$. The second guiding parameter, $\Lambda a^{2}$, changes, formally speaking, in the interval $-\infty<\Lambda a^{2}<\infty$.

a) When $\Lambda \leq 0$, the horizontal straight line $y=\Lambda a^{2}$ is below the graph of the function $y=f\left(\xi, \frac{\kappa}{a^{2}}\right)$, thus, there are no crossing. This means that $N(\xi)>0$ for arbitrary $\xi$, the metric has no horizons and the wormhole throat is traversable.

b) When $0<\Lambda a^{2}<f(0)$, there are two roots of the equation $N(\xi)=0$ (symmetrical with respect to the axis $\xi=0)$. The metric has two horizons, which can be indicated as the cosmological ones (see, e.g. [19]), since between them the $R$-region is situated with a traversable wormhole throat.

c) When $\Lambda a^{2}=f(0)$, we obtain that $N(0)=0$, and two horizons coincide, being situated at $r=0$. The wormhole throat is nontraversable.

d) When $\Lambda a^{2}>f(0)$, the straight line $y=\Lambda a^{2}$ lies above the graph of the function $y=f\left(\xi, \frac{\kappa}{a^{2}}\right)$. There is no crossing of these lines, and there are no horizons. Nevertheless, $N(\xi)<0$ for arbitrary $\xi$, i.e., now we cannot speak about a wormhole in the standard sense of the word.

\section{The second case: $2<\frac{\kappa}{a^{2}} \leq 4$}

For these values of the guiding parameter $\frac{\kappa}{a^{2}}$, the graph of the function $y=f\left(\xi, \frac{\kappa}{a^{2}}\right)$ lies at $y \geq 0$, it has two symmetric maxima, $f_{\max }^{*}$, and one minimum, at $\xi=0$, i.e., $f_{\min }=f(0) \geq 0$.

a) When $\Lambda a^{2}<0$, again the straight line $y=\Lambda a^{2}$ lies below the graph of the function $y=f\left(\xi, \frac{\kappa}{a^{2}}\right), N(\xi)>0$ for arbitrary $\xi$, the metric has no horizons, and the wormhole throat is traversable.

b) When $0<\Lambda a^{2}<f_{\min }=f(0)$, the equation $N(\xi)=0$ has two roots. Again there are two horizons of the cosmological type with intermediate $R$-region and the traversable throat.

c) When $\Lambda a^{2}=f(0)=0$, we obtain $N(0)=0$, i.e., the double horizon lies at $\xi=0$.

d) When $f_{\min }<\Lambda a^{2}<f_{\max }^{*}$, the straight line $y=\Lambda a^{2}$ crosses the graph of the auxiliary function in four points: $\xi= \pm \xi_{\text {in }}, \xi= \pm \xi_{\text {out }}$. Between pairs of horizons $\left(\xi_{\text {in }}<|\xi|<\xi_{\text {out }}\right)$ there exist two $R$-regions. The external horizons at $\xi= \pm \xi_{\text {out }}$ can be indicated as the cosmological ones, while the internal horizons at $\xi= \pm \xi_{\text {in }}$ are the event horizons.

e) When $\Lambda a^{2}=f_{\min }=f(0)$, two internal horizons coincide; effectively, there are three horizons, and the corresponding wormhole is nontraversable, since one (double) horizon is situated at $\xi=0$.

f) When $\Lambda a^{2}=f_{\max }^{*}$ the corresponding external and internal horizons coincide, so that two horizons appear instead of four; the wormhole is nontraversable.

g) When $\Lambda a^{2}>f_{\max }^{*}$, again $N(\xi)$ is negative everywhere.

\section{The third case: $\frac{\kappa}{a^{2}}>4$}

The graph of the auxiliary function has two symmetric maxima and one minimum at $\xi=0$, however, now the minimal value is negative, $f(0)<0$. Again, the

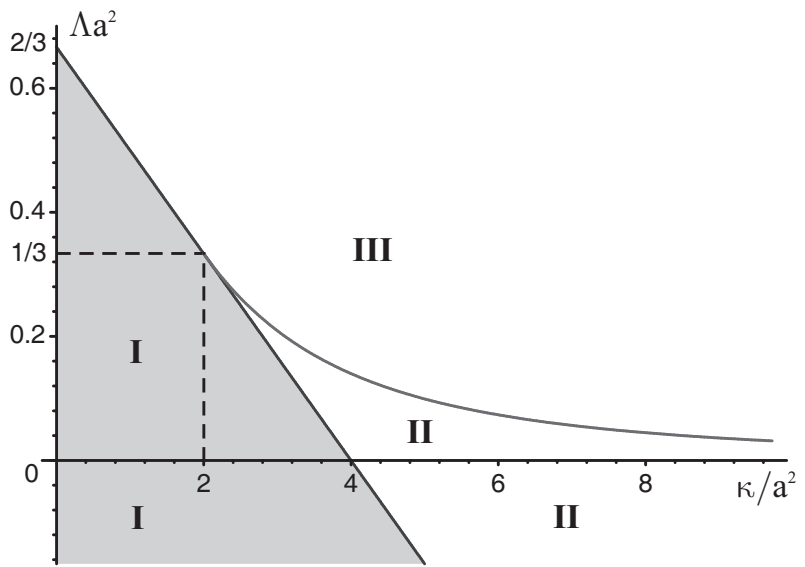

FIG. 2: Domains on the semiplane of the parameters $\frac{\kappa}{a^{2}}>0$ and $\Lambda a^{2}$, in which the $\Lambda$-influenced nonminimal Wu-Yang wormhole has a traversable or nontraversable throats. Domain I (shaded) indicates wormholes with a traversable throat, i.e., when $N(0)>0$. Domain II relates to the spacetimes with two $R$-regions and a $T$-region between them; the throat is nontraversable in this case. Domain III corresponds to the spacetimes without $R$-regions. The straight line $\Lambda a^{2}=f\left(0, \frac{\kappa}{a^{2}}\right)=\frac{2}{3}\left(1-\frac{\kappa}{4 a^{2}}\right)$ (dark) starts at $\frac{\kappa}{a^{2}}=0$ with $\Lambda a^{2}=\frac{2}{3}$. The curved line $\Lambda a^{2}=f_{\max }^{*}\left(\frac{\kappa}{a^{2}}\right)$ (grey) starts at $\frac{\kappa}{a^{2}}=2$ with $\Lambda a^{2}=\frac{1}{3}$; at $\kappa=2 a^{2}$, these two lines cross.

causal structure depends on the value of the guiding parameter $\Lambda a^{2}$.

a) When $\Lambda a^{2}<f(0)<0$, the straight line $y=\Lambda a^{2}$ is below the graph of the auxiliary function, and again $N(\xi)>0$ everywhere, thus, the wormhole throat is traversable.

b) When $\Lambda a^{2}=f(0)<0$, there is the double horizon in the throat.

c) When $f(0)<\Lambda a^{2} \leq 0$, the equation $N(\xi)=0$ has two symmetric roots. These roots correspond to the pair of the event horizons with a $T$-region between them.

d) When $0<\Lambda a^{2}<f_{\max }^{*}$, there are four crossing points: $\xi= \pm \xi_{\text {in }}, \xi= \pm \xi_{\text {out }}$. At $\xi_{\text {in }}<|\xi|<\xi_{\text {out }}$ there are $R$ regions, harbored by horizons. The external horizons can be indicated as the cosmological ones, while the internal horizons are the event ones.

e) When $\Lambda a^{2}=f_{\text {max }}^{*}$, the external and internal horizons coincide.

f) When $\Lambda a^{2}>f_{\max }^{*}$, the straight line $y=\Lambda a^{2}$ does not cross the graph of the function $y=f\left(\xi, \frac{\kappa}{a^{2}}\right)$; again, $N(\xi)<0$ for arbitrary $\xi$.

\section{Intermediate summary}

In order to summarize the results of the analysis we consider the plane of the parameters $\frac{\kappa}{a^{2}}, \Lambda a^{2}$, and indicate the domains without horizons, with one, two, three and four horizons. The results are illustrated in Fig. 2. 


\section{DISCUSSION}

In this paper, we discuss new exact solutions of the wormhole type obtained in the framework the model of nonminimal coupling between the gauge field of the $\mathrm{Wu}$ Yang type and gravity in a dark energy environment. The solutions of this model form a seven-parameter family: they depend generally on three nonminimal coupling constants $q_{1}, q_{2}, q_{3}$ (see (2) ), on two parameters $\omega_{\|}, \omega_{\perp}$, which appear in the equation of state for the dark energy (29), on the charge of the gauge field $\nu$ (see (21) ), entering the master equations for the gravity field via the parameter $\kappa \equiv 8 \pi \nu^{2}$, and on the initial value of the energy density of the dark energy $W_{0}$, which can be transformed into the effective cosmological constant in some special cases. In order to visualize exact solutions by presenting the metric function $\sigma(r)$ in the explicit form and the metric function $N(r)$ in quadratures, we considered various models with fixed parameters $\omega_{\|}$ and $\omega_{\perp}$. Along this line we discussed the solutions with $\omega_{\|}=-1$ and various $\omega_{\perp}$ (the model with anisotropic dark energy, see Subsection IVC); the solution with $\omega_{\perp}=\omega_{\|}=-\frac{1}{3}$ (analog of a string gas, see Subsection IVD); the solutions with $\omega_{\perp}=\omega_{\|}=-1$ (the model with effective cosmological constant $\Lambda=8 \pi W_{0}$, see Subsection IV C $(i)$ ).

Then, we extracted four-, three-, and twoparameter families of regular exact solutions from the mentioned four-parameter families by fixing the nonminimal coupling constants $q_{1}, q_{2}$ and $q_{3}$. In particular, when these constants are presented by equations (41), the first constitutive parameter is $\omega_{\|}=-1$ and the second one, $\omega_{\perp}$, is arbitrary; we find the four-parameter family of the wormhole-type solutions with $a$ describing the throat radius, $W_{0}$, connected with the initial value of the dark energy density (33), $\kappa=8 \pi \nu^{2}$, and $\omega_{\perp}$ (see Subsection IVC). When four guiding parameters of this anisotropic model, $a, W_{0}, \kappa$, and $\omega_{\perp}$, satisfy the following inequality

$$
1+4 \pi W_{0}\left(\omega_{\perp}-2\right) a^{2}>\frac{\kappa}{4 a^{2}},
$$

the throat of this wormhole is traversable, since the metric functions $\sigma(r)$ and $N(r)$ are regular, and $\sigma(0)>$ $0, N(0)>0$.

Another very interesting result is obtained for the isotropic distribution of the dark energy with $\omega_{\|}=$ $\omega_{\perp}=-\frac{1}{3}$ (this equation of state is known as a "string gas" [42]). If we assume that $q_{1}=0,4 q_{2}+q_{3}=0$ and $\frac{8 \pi}{3} W_{0} a^{2}+1=0$, the corresponding nonminimal wormhole is characterized by the catenary-type throat profile $R(r)=a \cosh \frac{r}{a}$, by the metric function $\sigma \equiv 1$ and by the function $N(r)$ presented explicitly by the polynomial of the fourth order of the variable $z=\tanh \frac{r}{a}$ (see formula (57)). The most illustrative regular solution of this type relates to the one-parameter model in which $C=0$ and the additional requirement (58) is used in order to fix the nonminimal coupling parameter $q_{2}$. This solution is of the form

$$
N(r)=\tanh ^{2} \frac{r}{a}+\frac{1}{4}\left(\frac{\kappa}{2 a^{2}}-1\right) \cosh ^{-4} \frac{r}{a},
$$

it has asymptotes $N( \pm \infty)=1$, and a finite value at the center $N(0)=\frac{1}{4}\left(\frac{\kappa}{2 a^{2}}-1\right)$. When $\kappa>2 a^{2}$, the presented function $N(r)$ has no zeroes, thus, this wormhole is traversable, and it links two regions of the spacetime with constant negative spatial curvature (or spatially open universes).

For the isotropic distribution of the dark energy with $\omega_{\|}=\omega_{\perp}=-1$, the solution (61), (62) describes a symmetric magnetic wormhole joining two asymptotically de Sitter (or asymptotically anti de Sitter) regions with the effective cosmological constant $\Lambda=8 \pi W_{0}$. This solution belongs to the same class of exact solutions describing nonminimal wormholes, which we obtained and discussed in [18] (magnetic wormholes joining two asymptotically Minkowski regions) and in [19] (electric wormholes joining an asymptotically Minkowski region and asymptotically de Sitter one). For this model, we considered in detail the problem of horizons, and have shown that, depending on the values of two effective guiding parameters $\frac{\kappa}{a^{2}}$ and $8 \pi W_{0} a^{2} \equiv \Lambda a^{2}$, the metric function $N(r)$ admits four, three, two, one zeroes, or does not admit zeroes at all. Correspondingly, there are two principally different wormhole configurations in the dark energy environment, which can be indicated as wormholes with traversable throats. First, the wormhole can have no horizons, and thus it is traversable in the general sense; this is possible when $\Lambda a^{2}<\frac{2}{3}\left(1-\frac{\kappa}{4 a^{2}}\right)$. Second, the wormhole can have two symmetric horizons, which are distant from the throat. The throat of such wormhole is traversable, and two horizons, in this sense, can be considered as the ones of a cosmological type. All other configurations have to be indicated as nontraversable. Indeed, when there is one zero of the metric function $N(r)$, or there are three zeroes, one horizon is inevitably situated at $r=0$, i.e., it appears just in the wormhole throat. When $N(r)$ has four zeroes, all four horizons are distant from the throat; nevertheless, this configuration has to be indicated as the nontraversable wormhole, since, in addition to the pair of cosmological horizons, two distant event horizons, which close the entrance to the throat and the exit from it, appear.

To conclude, we have to emphasize two new aspects of the obtained results.

First, we have shown that the dark energy influence can effectively regulate the traversability of the nonminimal $\mathrm{Wu}$-Yang wormholes: it can create the horizon just inside the throat, and it can organize two distant horizons at the entrance and exit of the throat. On the other hand, the dark energy can open the nonminimal wormhole throat for traveling from one region of the spacetime to another. For instance, when we deal with the dark energy of the $\Lambda$-type, we can distinguish two situations: for the case $\Lambda=0$ and $\frac{\kappa}{4 a^{2}}>1$, the wormhole is nontraversable; when $\Lambda \neq 0$ and $\Lambda a^{2}<\frac{2}{3}\left(1-\frac{\kappa}{4 a^{2}}\right)$, the entrance to the wormhole throat happens to be opened. When the dark energy pressure is not constant, using the $t$-representation of the model, we could try to find specific epochs in the Universe history for which the cosmic gates related to fixed gauge charge $\nu$ are opened or closed; we hope to discuss this problem in the future.

Second, our study allows us to view from a new perspective a situation with the number of fundamental 
constants. The problem can be formulated as follows: is at least one nonminimal coupling constant (e.g., $q_{1}$ ) a new constant of Nature, or should all three parameters, $q_{1}, q_{2}$, and $q_{3}$, be reduced to the combinations of already-known fundamental constants? Our study gives food for thought in this relation. One can assume that the nonminimal coupling constants can be connected with parameters of the dark energy. For instance, if we assume that $\Lambda$ is negative (it is typical for anti-de Sitter asymptotes) and require that the value $N(0)$ of the metric function $N(r)$ (see (63) ) is positive and does not depend on the value of the gauge charge $\nu$, we obtain that $q_{1}=-\frac{1}{6 \Lambda}$ and $N(0)=\frac{1}{3}>0$. In other words, the key nonminimal coupling constant is not independent and is reciprocal to the cosmological constant. Clearly, this relationship is not unique, and we hope to discuss a few interesting ideas in the next work.

\section{Acknowledgments}

This work was partially supported by the Russian Foundation for Basic Research (Grant No. 1402-00598), and by the Program of Competitive Growth of Kazan Federal University (Project No. 0615/006.15.02302.034).
[1] E.J. Copeland, M. Sami, and S. Tsujikawa, Int. J. Mod. Phys. D 15, 1753 (2006).

[2] J. Frieman, M. Turner, and D. Huterer, Ann. Rev. Astron. Astrophys. 46, 385 (2008).

[3] T. Padmanabhan, Gen. Relativ. Gravit. 40, 529 (2007).

[4] B. Saha, Phys. Part. Nucl. 45, 349 (2014).

[5] S.J. Perlmutter et al., Nature 391, 51 (1998).

[6] A.G. Riess et al., Astron. J. 116, 1009 (1998).

[7] B.P. Schmidt et al., Astrophys. J. 507, 46 (1998).

[8] S. Nojiri and S. D. Odintsov, Phys. Rep. 505, 59 (2011).

[9] G.R. Farrar and P.J.E. Peebles, Astrophys. J. 604, 1 (2004).

[10] B. Wang, C.-Y. Lin, D. Pavon, and E. Abdalla, Phys. Lett. B 662, 1 (2008).

[11] A. Pourtsidou, C. Skordis, and E.J. Copeland, Phys. Rev. D 88, 083505 (2013).

[12] A.B. Balakin and V.V. Bochkarev, Phys. Rev. D 83, 024035 (2011);

A.B. Balakin and V.V. Bochkarev, Phys. Rev. D 83, 024036 (2011);

A.B. Balakin and V.V. Bochkarev, Phys. Rev. D 87, 024006 (2013).

[13] A.B. Balakin and N.N. Dolbilova, Phys. Rev. D 89, $104012(2014)$

[14] G. W. Horndeski, Arch. Rat. Mech. Anal. 75, 229 (1981).

[15] F. Müller-Hoissen, Classical Quantum Gravity 5, L35 (1988).

[16] A.B. Balakin and A.E. Zayats, Gravit. Cosmol. 12, 302 (2006)

[17] A.B. Balakin and A.E. Zayats, Phys. Lett. B 644, 294 (2007).

[18] A.B. Balakin, S.V. Sushkov, and A.E. Zayats, Phys. Rev. D 75, 084042 (2007).

[19] A.B. Balakin, J.P.S. Lemos, and A.E. Zayats, Phys. Rev. D 81, 084015 (2010).

[20] S.-W. Kim, Phys. Lett. A 166, 13 (1992).

[21] L.-X. Li, J. Geom. Phys. 40, 154 (2001).

[22] J. P. S. Lemos, F. S. N. Lobo, and S. Q. de Oliveira, Phys. Rev. D 68, 064004 (2003).

[23] V. Rubakov, Classical Theory of Gauge Fields (Princeton University Press, Princeton and Oxford, 2002).

[24] A. R. Prasanna, Phys. Lett. A 37, 331 (1971).

[25] I. T. Drummond and S. J. Hathrell, Phys. Rev. D 22, 343 (1980).
[26] A. B. Balakin and J. P. S. Lemos, Class. Quantum Grav. 22, 1867 (2005).

[27] H. Stephani, D. Kramer, M.A.H. MacCallum, C. Hoenselaers, and E. Herlt, Exact Solutions of Einstein's Field Equations (Cambridge University Press, Cambridge, England, 2003).

[28] A. C. Eringen and G. A. Maugin, Electrodynamics of Continua (Springer-Verlag, New York, 1989).

[29] F. W. Hehl and Yu. N. Obukhov, Foundations of Classical Electrodynamics: Charge, Flux, and Metric (Birkhaüser, Boston, 2003).

[30] A. I. Akhiezer and S. V. Peletminsky, Fields and Fundamental Interactions (Taylor and Francis, London, 2002).

[31] M.S. Morris and K.S. Thorne, Am. J. Phys. 56, 395 (1988).

[32] D. Hochberg and M. Visser, Phys. Rev. D 56, 4745 (1997);

D. Hochberg and M. Visser, Phys. Rev. D 58, 044021 (1998).

[33] M. Visser, Lorentzian Wormholes: From Einstein to Hawking (AIP Press, New York, 1995).

[34] F.S.N. Lobo, in Classical and Quantum Gravity Research, edited by C.N. Mikkel and T.K. Rasmussen (Nova Science, New York, 2008), p. 1.

[35] H.G. Ellis, J. Math. Phys. (N.Y.) 14, 104 (1973).

[36] K.A. Bronnikov, Acta Phys. Pol. B 4, 251 (1973).

[37] C. Barceló and M. Visser, Phys. Lett. B 466, 127 (1999);

C. Barceló and M. Visser, Class. Quantum Gravity 17, 3843 (2000).

[38] O. Hauser, R. Ibadov, B. Kleihaus, and J. Kunz, Phys. Rev. D 89, 064010 (2014).

[39] T. Bandyopadhyay and S. Chakraborty, Class. Quantum Grav. 26, 085005 (2009);

S. Habib Mazharimousavi, M. Halilsoy, and Z. Amirabi, Class. Quantum Grav. 28, 025004 (2011).

[40] T. T. Wu and C. N. Yang, in Properties of Matter Under Unusual Conditions, edited by H. Mark and S. Fernbach (Interscience, New York, 1969), p. 349.

[41] F. A. Bais, Phys. Lett. B 64, 465 (1976).

[42] A. Yu. Kamenshchik and I. M. Khalatnikov, Int. J. Mod. Phys. D 21, 1250004 (2012). 